\title{
AVALIAÇÃO DA QUALIDADE DE MUDAS DE Eucalyptus grandis UTILIZANDO PARÂMETROS MORFOLÓGICOS
}

\author{
Elder Eloy ${ }^{1}$, Braulio Otomar Caron², Denise Schmidt², Alexandre Behling ${ }^{3}$, Luciano Schwers ${ }^{4}$, \\ Elvis Felipe Elli ${ }^{5}$ \\ ${ }^{1}$ Eng. Florestal, Doutorando em Engenharia Florestal, UFPR, Curitiba, PR, Brasil - eloyelder@yahoo.com.br \\ ${ }^{2}$ Eng. Agrônomo, Dr., Depto. de Agronomia, UFSM/CESNORS, Frederico Westphalen, RS, Brasil - otomarcaron@ yahoo.com.br - \\ denises@smail.ufsm.br \\ ${ }^{3}$ Eng. Florestal, Mestrando em Engenharia Florestal, UFPR, Curitiba, PR, Brasil - alexandre.behling @ yahoo.com.br \\ ${ }^{4}$ Eng. Agrônomo, Doutorando em Agronomia USP/ESALQ, Piracicaba, SP, Brasil - luagronomia@ hotmail.com \\ ${ }^{5}$ Eng. Agrônomo, Depto. de Agronomia, UFSM/CESNORS, Frederico Westphalen, RS, Brasil - elvisfelipeelli@yahoo.com
}

Recebido para publicação: 04/05/2012 - Aceito para publicação: 18/06/2013

\begin{abstract}
O presente trabalho teve como objetivo determinar o índice de qualidade Dickson (IQD) e a sua relação com as variáveis morfológicas em mudas de Eucalyptus grandis W. Hill ex Maiden produzidas em diferentes tratamentos. O experimento foi conduzido no viveiro agronômico do Centro de Educação Superior Norte do Rio Grande do Sul (CESNORS), em delineamento experimental de blocos completamente casualizados. Foram testados quatro tratamentos, que envolveram dois volumes de tubetes $\left(50\right.$ e $90 \mathrm{~cm}^{3}$ ) e dois níveis de densidade de plantas na bandeja. Avaliaram-se altura da parte aérea, diâmetro do colo, massa seca de folhas, massa seca do caule, massa seca da parte aérea, massa seca de raiz, massa seca total (MST), área foliar (AF) e IQD. O tamanho do tubete e a densidade das mudas na bandeja influenciam significativamente no crescimento das mudas ao longo do tempo, limitando o volume de substrato explorável e gerando competição por espaço pelas plantas. Todas as variáveis avaliadas se correlacionaram significativamente, sendo que o IQD demonstrou ter alto índice com a MST. Os tratamentos com recipiente de volume $90 \mathrm{~cm}^{3}$ foram os que apresentaram os melhores IQD, resultando em mudas de boa qualidade, capazes de suportar as condições adversas do campo.

Palavras-chave: Eucalipto; tubete; densidade de mudas; índice de qualidade de Dickson.
\end{abstract}

Resumo

\begin{abstract}
Quality assessment of Eucalyptus grandis seedlings using morphological parameters. The present work had as objective determines the index of Dickson's quality (IDQ) and its relation to the morphologic variables in Eucalyptus grandis W. Hill ex Maiden seedlings produced in different treatments. The experiment was driven in the agronomic nursery of the Center of Superior Education North of Rio Grande do Sul (CESNORS), in experimental design of randomized complete block. Four treatments were tested, that involved two volumes sizes $\left(50\right.$ and $\left.90 \mathrm{~cm}^{3}\right)$ and two levels of density of plants in the tray. It evaluated height of the aerial part, diameter of the lap, mass dries of leaves, mass dries of the stem, mass dries of the aerial part, mass dries of root, mass total drought (MST), foliar area (AF) and the index of Dickson's quality (IDQ). The size of the containers and the density of the seedlings in the tray had significant influence in growth of the seedlings along the time, putting limits to the volume of exploitable substratum and generating competition for space by the plants. All the appraised variables correlated significantly and IDQ revealed high index with MST. The treatments with container of volume $90 \mathrm{~cm}^{3}$ were the ones that presented the best IQD, resulting in seedlings of good quality capable to support the adverse conditions of the field.

Keywords: Eucalyptus; containers; density of seedlings; index of Dickson quality.
\end{abstract}

\section{INTRODUÇÃO}

Atualmente, o aumento no consumo de madeira e seus derivados, tanto na área energética quanto na de beneficiamento e de transformação, evidencia a necessidade de geração de novas tecnologias de 
produção de mudas, com um padrão de qualidade adequado, visando o estabelecimento de florestas cada vez mais produtivas.

A expansão na demanda por produtos florestais tem como consequência direta a necessidade de introduzir, nos programas de reflorestamento no Brasil, espécies de alta produtividade, que permitam reduzir relativamente o ciclo de corte, associada às boas características silviculturais aplicadas, disponibilizando para as mudas uma maior resistência às condições adversas do meio e um menor tempo gasto para a sua completa formação, sendo tais fatores decisivos ao seu êxito (CRUZ et al., 2004).

Na seleção de mudas para o plantio, são utilizados critérios baseados em características que, na maioria das vezes, não determinam as reais qualidades, visto que elas variam de acordo com a espécie, sítios ecológicos, tratos culturais, transporte, distribuição e plantio das mudas. Assim, existem várias razões para a utilização de testes na definição do padrão de qualidade de mudas, podendo-se agregar alguns valores que, muitas vezes, são exigidos pelo mercado (GOMES et al., 2002).

$\mathrm{Na}$ determinação da qualidade das mudas, podem ser utilizadas características tanto morfológicas, que são baseadas nos aspectos fenotípicos, quanto fisiológicas, que são definidas internamente na planta. As características morfológicas ainda são as mais utilizadas para determinar qualidade das mudas, pois têm maior aceitação dos viveiristas. Porém ainda carece de definições que possam responder à sobrevivência e ao crescimento inicial, em função das adversidades que são encontradas em campo após o plantio.

O índice de qualidade de Dickson é mencionado como uma promissora medida morfológica integrada e apontado como bom indicador da qualidade de mudas, por considerar para o seu cálculo a robustez e o equilíbrio da distribuição da fitomassa, sendo ponderados vários parâmetros importantes. As observações dos parâmetros morfológicos apresentam, além de uma boa qualificação, atributos de fácil aplicação física e visíveis na planta, que podem ser alcançados de maneira prática e rápida, sendo muito utilizados para estabelecer o momento da realização de atividades silviculturais, importantes para obtenção de mudas com melhor qualidade (FONSECA et al., 2002).

A sofisticação das técnicas utilizadas visa à obtenção de um padrão de qualidade adequado das mudas a serem produzidas e, consequentemente, aumento da produtividade do povoamento florestal. Assim, pesquisadores têm procurado definir as melhores metodologias, recipientes, substratos e fertilizações para a produção de mudas florestais que apresentam altas taxas de sobrevivência e desempenho após o plantio.

O uso de recipientes inadequados é uma das causas mais comuns da malformação do sistema radicular das mudas no viveiro, que promovem a distribuição anormal de raízes laterais e superficiais, ocasionando o desequilíbrio na absorção de água e nutrientes em quantidades suficientes para atender às necessidades da planta (MAFIA et al., 2005). Isso resulta em um quadro sintomatológico típico de deficiência hídrica ou nutricional, em consequência do desequilíbrio entre raiz e parte aérea.

A densidade das mudas no viveiro é um fator importante que gera grande influência no crescimento e no desenvolvimento da muda, mas ainda carece de estudos relacionados à produção de mudas em tubetes na fase de pré-viveiro. De modo geral, entende-se que o espaçamento entre os tubetes na bandeja permite, além da seleção e remoção das mudas em formação, otimizar espaço no ambiente de cultivo, reduzindo consideravelmente os custos de manutenção, principalmente de mão de obra, que está diretamente envolvida nessa fase da produção (TEIXEIRA, 2009). Para Reis et al. (2008), um melhor arranjo entre as plantas proporciona uma distribuição mais uniforme do crescimento, tornando-o mais homogêneo e, consequentemente, apresentando um melhor padrão de qualidade.

Nesse contexto, o presente trabalho visa à obtenção de mudas com boa qualidade. Assim, este trabalho teve como intuito determinar o índice de qualidade Dickson e a sua relação com as variáveis morfológicas em mudas de Eucalyptus grandis W. Hill ex Maiden distribuídas em tubetes com diferentes volumes e diferentes densidades de mudas nas bandejas, em casa de vegetação.

\section{MATERIAIS E MÉTODOS}

O experimento foi realizado no período de agosto de 2008 a janeiro de 2009, no viveiro do Departamento de Agronomia vinculado ao Laboratório de Agroclimatologia da Universidade Federal de Santa Maria, Campi CESNORS (Centro de Educação Superior Norte do Rio Grande do Sul), sob 
coordenadas geográficas de $27^{\circ} 22^{\prime} \mathrm{S}, 53^{\circ} 25^{\prime} \mathrm{W}$, a $480 \mathrm{~m}$ de altitude, no município de Frederico Westphalen (RS).

De acordo com a classificação climática de Köppen, o clima da região é Cfb, ou seja, subtropical úmido com temperatura média anual de $19,1{ }^{\circ} \mathrm{C}$, variando com máxima de $38{ }^{\circ} \mathrm{C}$ e mínima de $0{ }^{\circ} \mathrm{C}$, apresentando uma precipitação média anual de $1606 \mathrm{~mm}$.

As sementes de Eucalyptus grandis foram produzidas pela empresa Klabin no município de Telêmaco Borba (PR). A semeadura foi realizada em 24 de agosto de 2008 diretamente nos tubetes cônicos com seis estrias de polipropileno de fundo aberto, preenchidos com substrato constituído de material orgânico $\left(\operatorname{Tecnomax}^{\circledR}\right)$, sem adubação de base, acondicionados em bandejas plásticas, colocando-se em média 10 sementes em cada tubete.

Após as plântulas completarem 15 dias, foi efetuado o raleio, com o objetivo de eliminar as excedentes em cada recipiente, deixando apenas a que apresentava maior qualidade e posição mais central. Durante a realização do experimento, a irrigação foi realizada diariamente, de forma manual, mantendo sempre a capacidade de campo do substrato para todos os tratamentos.

O experimento foi instalado utilizando-se o delineamento experimental de blocos completamente casualizados com $2 \times 2$ tratamentos fatoriais, ou seja, dois tamanhos de tubetes $(\mathrm{TP}=$ Tamanho Pequeno $=$ $50 \mathrm{~cm}^{3}$ e TM = tamanho médio $=90 \mathrm{~cm}^{3}$ ) e dois níveis de densidade (DA = Densidade Alta, ocupação de todas as células da bandeja $=736$ e 528 plantas. $\mathrm{m}^{-2}\left(\mathrm{p} / \mathrm{m}^{2}\right)$ nas bandejas com os tubetes de $50 \mathrm{e} 90 \mathrm{~cm}^{3}$, respectivamente, e DM = densidade média $=368$ e $264 \mathrm{p} / \mathrm{m}^{2}$ nas bandejas com tubetes com 50 e $90 \mathrm{~cm}^{3}$, respectivamente). Isso resultou na seguinte notação de tratamentos aplicados nas parcelas: TPDA, TPDM, TMDA e TMDM, sendo o segundo considerado o tratamento controle. O bloco contemplou quatro unidades experimentais nas quais foram distribuídas os dois tamanhos de tubetes e as duas densidades de mudas na bandeja. As unidades experimentais contemplaram 66 e 90 plantas para DA e DM, respectivamente, sendo as mesmas divididas nas sete avaliações realizadas ao longo do tempo, compostas de duas mudas contíguas internas com seis repetições, utilizando-se bordadura dupla.

As avaliações foram realizadas ao longo do tempo a cada 15 dias, sendo a primeira avaliada a partir dos 50 dias após a emergência (DAE) e a última aos 140 DAE. Nas sete avaliações realizadas, foram avaliadas as seguintes características morfológicas: altura da parte aérea $(\mathrm{H})$, diâmetro do colo (DC), massa seca de folhas (MSF), massa seca do caule (MSC), massa seca da parte aérea (MSPA), massa seca de raiz (MSR), massa seca total (MST), área foliar (AF) e índice de qualidade de Dickson (IQD).

A $\mathrm{H}$ foi determinada a partir do nível do substrato até a ponta da última folha, com o auxílio de régua graduada em milímetros, e o DC foi mensurado no nível do substrato, com o auxílio de paquímetro digital, com precisão de milímetro. As determinações dos MSPA e da MSR foram efetuadas a partir da separação da parte aérea e radicular da planta, com posterior secagem dos materiais em estufa com ventilação forçada a $65{ }^{\circ} \mathrm{C}$ até peso constante, sendo a pesagem realizada com o auxílio de balança eletrônica, com precisão de miligramas. A MST foi obtida através da soma das MSPA e da MSR. As relações entre as características medidas foram determinadas pela simples divisão entre elas.

A área foliar foi obtida através do uso do seguinte modelo proposto por Caron et al. (2012):

$$
\mathrm{AF}=\left[\left(\mathrm{c}_{1} \times \mathrm{l}_{1}\right) \times 0,7226\right] \times \mathrm{nfp}+\left[\left(\mathrm{c}_{2} \times \mathrm{l}_{2}\right) \times 0,7226\right] \times \mathrm{nfm}+\left[\left(\mathrm{c}_{3} \times \mathrm{l}_{3}\right) \times 0,7226\right] \times \mathrm{nfg}
$$

Em que: $\mathrm{AF}=$ área foliar, $\mathrm{em} \mathrm{cm}^{2} ; \mathrm{c}_{1}=$ comprimento mediano da folha de tamanho pequeno, $\mathrm{em} \mathrm{cm}$; $\mathrm{c}_{2}=$ comprimento mediano da folha de tamanho médio, em $\mathrm{cm} ; \mathrm{c}_{3}=$ comprimento mediano da folha de tamanho grande, em $\mathrm{cm} ; 1_{1}=$ largura mediana da folha de tamanho pequeno, em $\mathrm{cm} ; 1_{2}=$ largura mediana da folha de tamanho médio, em $\mathrm{cm} ; 1_{3}=$ largura mediana da folha de tamanho grande, em $\mathrm{cm}$; $\mathrm{nfp}=$ número total de folhas pequenas (até $2,5 \mathrm{~cm}$ de comprimento); $\mathrm{nfm}=$ número total de folhas médias (de 2,6 a $5 \mathrm{~cm}$ de comprimento); $\mathrm{nfg}=$ número total de folhas grandes (maior que $5 \mathrm{~cm}$ de comprimento); e 0,7226 = fator de correção do retângulo (comprimento x largura da folha).

O IQD foi determinado em função de H, DC, MSPA e MSR, por meio da seguinte fórmula (DICKSON et al., 1960):

$$
\mathrm{IQD}=\frac{\text { MST g }}{\frac{\mathrm{H} \mathrm{cm}}{\mathrm{DC} \mathrm{mm}}+\frac{\text { MSPA g }}{\text { MSR g }}}
$$


Em que: MST = massa seca total, em g; MSPA = massa seca da parte aérea, em g; MSR = massa seca de raiz, em g; $\mathrm{H}=$ altura da parte aérea, em cm; DC = diâmetro do colo, em mm.

Os dados obtidos foram submetidos à análise estatística através do software SAS Learning Edition 8.0 (2003), em que se determinou a análise de variância, a análise de regressão, a análise de correlação de Pearson, o teste F e o teste de Tukey a 5\% de probabilidade.

\section{RESULTADOS E DISCUSSÃO}

A análise de variância apontou diferença significativa para altura, diâmetro de colo, massa seca da folha, massa seca do caule, massa seca de raiz, massa seca total, área foliar e índice de qualidade de Dickson para as variáveis dias após a emergência (50,65, 80, 95, 110, 125 e 140 dias) e tratamentos (TPDA, TPDM, TMDA e TMDM). Essa característica não foi observada para o diâmetro de colo e massa seca de raiz na interação dias após a emergência $x$ tratamento. Observou-se diferença significativa em relação aos diferentes tratamentos estudados a partir de 65 dias após a germinação (DAE) para diâmetro de colo e índice de qualidade Dickson. Já para as variáveis altura, massa seca total e área foliar foi observada a partir dos 80 DAE, e após os 90 DAE para massa seca do caule (Tabela 1).

Tabela 1. Análise de variância para altura (H), diâmetro do colo (DC), massa seca de folhas (MSF), massa seca do caule (MSC), massa seca de raiz (MSR), massa seca total (MST), área foliar (AF) e índice de qualidade de Dickson (IQD) em diferentes dias após a emergência (DAE), nos quatro tratamentos (TRAT) testados, em mudas de Eucalyptus grandis.

Table 1. Analysis of variance for height (H), diameter (DC), leaf dry mass (MSF), stem dry mass (MSC), root dry weight (MSR), total dry matter (MST), leaf area (AF) and quality index of Dickson (IQD) on different days after emergence (DAE), the four treatments (TRAT) tested in Eucalyptus grandis.

\begin{tabular}{|c|c|c|c|c|c|c|c|c|c|}
\hline \multicolumn{10}{|c|}{ Efeito principal } \\
\hline \multirow{2}{*}{ Fator de estudo } & \multirow{2}{*}{ GL } & \multicolumn{8}{|c|}{ Quadrado médio } \\
\hline & & $\mathbf{H}$ & DC & MSF & MSC & MSR & MST & $\mathbf{A F}$ & IQD \\
\hline DAE & 6 & $8426,84 *$ & $21,19^{*}$ & $22,97 *$ & $23,93^{*}$ & $9,19 *$ & $158,65^{*}$ & $250270 *$ & $0,35^{*}$ \\
\hline TRAT & 3 & $1012,11 *$ & $2,38^{*}$ & $9,05^{*}$ & $4,56^{*}$ & $3,32 *$ & $36,69 *$ & 101979* & $0,18^{*}$ \\
\hline DAE $x$ TRAT & 18 & $50,85^{*}$ & $0,08^{\mathrm{ns}}$ & $0,61 *$ & $1,02 *$ & $0,16^{\mathrm{ns}}$ & $6,99 *$ & $8204 *$ & $0,01 *$ \\
\hline $\mathrm{R}^{2}$ & & 0,95 & 0,91 & 0,88 & 0,81 & 0,83 & 0,72 & 0,79 & 0,77 \\
\hline $\mathrm{CV}(\%)$ & & 14,36 & 12,35 & 25,36 & 50,81 & 27,35 & 46,20 & 39,61 & 30,39 \\
\hline \multicolumn{10}{|c|}{ Efeito simples } \\
\hline \multicolumn{10}{|c|}{ DAG x TRAT } \\
\hline \multirow[t]{7}{*}{ DAE } & 50 & $13,83^{\mathrm{ns}}$ & - & $0,10^{\mathrm{ns}}$ & $0,01^{\mathrm{ns}}$ & - & $0,43^{\mathrm{ns}}$ & $793,75^{\mathrm{ns}}$ & $0,01^{\mathrm{ns}}$ \\
\hline & 65 & $34,51^{\mathrm{ns}}$ & - & $0,21^{\mathrm{ns}}$ & $0,01^{\mathrm{ns}}$ & - & $0,83^{\mathrm{ns}}$ & $2718,46^{\mathrm{ns}}$ & $0,01^{\mathrm{ns}}$ \\
\hline & 30 & $48,01^{\mathrm{ns}}$ & - & $0,83^{*}$ & $0,05^{\mathrm{ns}}$ & - & $2,35^{\mathrm{ns}}$ & $5725,97^{\mathrm{ns}}$ & $0,02 *$ \\
\hline & 95 & $256,52 *$ & - & $0,83^{*}$ & $0,07^{\mathrm{ns}}$ & - & $8,94 *$ & $16397 *$ & $0,03^{*}$ \\
\hline & 10 & $237,33^{*}$ & - & $1,99 *$ & $0,93^{*}$ & - & $8,33^{*}$ & $29794 *$ & $0,03 *$ \\
\hline & 25 & $346,08 *$ & - & $4,17^{*}$ & $2,21 *$ & - & $21,16^{*}$ & $46934 *$ & $0,07 *$ \\
\hline & 40 & $381,28 *$ & - & $4,58^{*}$ & $7,48^{*}$ & - & $36,59 *$ & $51991 *$ & $0,11^{*}$ \\
\hline \multirow[t]{4}{*}{ TRAT } & DA & $2175,45^{*}$ & - & $3,04 *$ & $3,24 *$ & - & $22,67 *$ & $30602 *$ & $0,04^{*}$ \\
\hline & $\mathrm{DM}$ & $1500,03^{*}$ & - & $3,34 *$ & $2,19 *$ & - & $28,12 *$ & $38858^{*}$ & $0,07^{*}$ \\
\hline & IDA & $2895,79 *$ & - & $8,34^{*}$ & $12,33^{*}$ & - & $66,24 *$ & $100104 *$ & $0,13^{*}$ \\
\hline & IDM & $2008,11 *$ & - & $10,09^{*}$ & $9,24 *$ & - & $62,59 *$ & $105949 *$ & $0,15^{*}$ \\
\hline
\end{tabular}

*: significativo a 5\% de probabilidade; ns: não significativo a 5\% de probabilidade; TPDA: tubete pequeno + densidade alta; TPDM: tubete pequeno + densidade média; TMDA: tubete médio + densidade alta; TMDM: tubete médio + densidade média.

Os quatro tratamentos estudados promoveram crescimentos estatisticamente diferentes, para todas as variáveis nas sete idades avaliadas. Este resultado foi o esperado, uma vez que o tubete de maior volume $\left(90 \mathrm{~cm}^{3}\right)$ é quase o dobro do menor $\left(50 \mathrm{~cm}^{3}\right)$, e a quantidade de mudas presentes na bandeja de densidade alta é o dobro da quantidade presente na de densidade média. 
Para a variável altura, observaram-se os maiores valores médios para o tratamento TMDA (66,04 cm aos 140 dias), que apresenta maior volume de recipiente e alta densidade de mudas na bandeja, isso motivado pelo maior volume de substrato explorável disponível para as plantas, o que promoveu um maior crescimento das raízes, além de que a maior densidade de plantas influenciou na competição por espaço, induzindo a um maior crescimento. Esse fato foi também verificado para a área foliar e a massa seca total aos 140 DAE. Da mesma forma, nos tratamentos que apresentavam tubetes com $50 \mathrm{~cm}^{3}$, observou-se crescimento significativamente menor (Tabela 2 e Figura 1).

Tabela 2. Teste de médias para as variáveis altura $(\mathrm{H})$, em $\mathrm{cm}$, diâmetro do colo (DC), em mm, massa seca de folhas (MSF), em g, massa seca do caule (MSC), em g, massa seca de raiz (MSR), em g, massa seca total (MST), em g, área foliar (AF), em $\mathrm{cm}^{2}$, e índice de qualidade de Dickson (IQD) em diferentes dias após a emergência (DAE), nos quatro tratamentos (TRAT) testados, em mudas de Eucalyptus grandis.

Table 2. Test averages for the variables height $(\mathrm{H})$, in $\mathrm{cm}$, diameter (DC), in mm, leaf dry mass (MSF), in $\mathrm{g}$, stem dry weight (MSC), in g, mass dry root (MSR), in g, total dry weight (MST), in g, leaf area (AF), in $\mathrm{cm}^{2}$, and quality index of Dickson (IQD) on different days after emergence (DAE), in four treatments (TRAT) tested in Eucalyptus grandis.

\begin{tabular}{|c|c|c|c|c|c|c|c|c|c|}
\hline DAE & TRAT & $\mathbf{H}$ & DC & MSF & MSC & MSR & MST & $\mathbf{A F}$ & IQD \\
\hline \multirow[t]{4}{*}{50} & TPDA & $9,16^{\mathrm{ns}}$ & $1,18^{\mathrm{ns}}$ & $0,25^{\mathrm{ns}}$ & $0,08^{\mathrm{ns}}$ & $0,24^{\mathrm{ns}}$ & $0,57^{\mathrm{ns}}$ & $19,04^{\mathrm{ns}}$ & $0,06^{\mathrm{ns}}$ \\
\hline & TPDM & $8,25^{\mathrm{ns}}$ & $1,12^{\mathrm{ns}}$ & $0,25^{\mathrm{ns}}$ & $0,06^{\mathrm{ns}}$ & $0,23^{\mathrm{ns}}$ & $0,53^{\mathrm{ns}}$ & $18,89^{\mathrm{ns}}$ & $0,06^{\mathrm{ns}}$ \\
\hline & TMDA & $11,73^{\mathrm{ns}}$ & $1,39^{\mathrm{ns}}$ & $0,47^{\mathrm{ns}}$ & $0,12^{\mathrm{ns}}$ & $0,40^{\mathrm{ns}}$ & $0,99^{\mathrm{ns}}$ & $41,77^{\mathrm{ns}}$ & $0,10^{\mathrm{ns}}$ \\
\hline & TMDM & $10,46^{\mathrm{ns}}$ & $1,43^{\mathrm{ns}}$ & $0,48^{\mathrm{ns}}$ & $0,12^{\mathrm{ns}}$ & $0,44^{\mathrm{ns}}$ & $1,04^{\mathrm{ns}}$ & $34,75^{\mathrm{ns}}$ & $0,12^{\mathrm{ns}}$ \\
\hline \multirow[t]{4}{*}{65} & TPDA & $12,43^{\mathrm{ns}}$ & $1,46^{\mathrm{ns}}$ & $0,46^{\mathrm{ns}}$ & $0,12^{\mathrm{ns}}$ & $0,47^{\mathrm{ns}}$ & $1,05^{\mathrm{ns}}$ & $43,29^{\mathrm{ns}}$ & $0,11^{\mathrm{ns}}$ \\
\hline & TPDM & $10,67^{\mathrm{ns}}$ & $1,49^{\mathrm{ns}}$ & $0,60^{\mathrm{ns}}$ & $0,15^{\mathrm{ns}}$ & $0,38^{\mathrm{ns}}$ & $1,14^{\mathrm{ns}}$ & $46,88^{\mathrm{ns}}$ & $0,12^{\mathrm{ns}}$ \\
\hline & TMDA & $16,29^{\mathrm{ns}}$ & $1,73^{\mathrm{ns}}$ & $0,77^{\mathrm{ns}}$ & $0,22^{\mathrm{ns}}$ & $0,64^{\mathrm{ns}}$ & $1,63^{\mathrm{ns}}$ & $84,15^{\mathrm{ns}}$ & $0,15^{\mathrm{ns}}$ \\
\hline & TMDM & $14,09^{\mathrm{ns}}$ & $1,75^{\mathrm{ns}}$ & $0,89^{\mathrm{ns}}$ & $0,19^{\mathrm{ns}}$ & $0,74^{\mathrm{ns}}$ & $1,82^{\mathrm{ns}}$ & $79,26^{\mathrm{ns}}$ & $0,20^{\mathrm{ns}}$ \\
\hline \multirow[t]{4}{*}{80} & TPDA & $18,28^{\mathrm{ns}}$ & $1,77 \mathrm{~b}$ & $0,65 \mathrm{~b}$ & $0,42^{\mathrm{ns}}$ & $0,73 \mathrm{~b}$ & $1,80^{\mathrm{ns}}$ & $66,98^{\mathrm{ns}}$ & $0,15 \mathrm{~b}$ \\
\hline & TPDM & $16,18^{\mathrm{ns}}$ & $1,80 \mathrm{~b}$ & $0,84 \mathrm{~b}$ & $0,26^{\mathrm{ns}}$ & $0,71 \mathrm{~b}$ & $1,81^{\mathrm{ns}}$ & $57,43^{\mathrm{ns}}$ & $0,17 \mathrm{ab}$ \\
\hline & TMDA & $22,94^{\mathrm{ns}}$ & $2,21 \mathrm{a}$ & $1,51 \mathrm{a}$ & $0,43^{\mathrm{ns}}$ & $1,13 \mathrm{a}$ & $3,07^{\mathrm{ns}}$ & $126,37^{\mathrm{ns}}$ & $0,25 \mathrm{a}$ \\
\hline & TMDM & $18,84^{\mathrm{ns}}$ & $2,13 \mathrm{ab}$ & $1,08 \mathrm{ab}$ & $0,30^{\mathrm{ns}}$ & $1,21 \mathrm{a}$ & $2,60^{\mathrm{ns}}$ & $93,42^{\mathrm{ns}}$ & $0,26 \mathrm{a}$ \\
\hline \multirow[t]{4}{*}{95} & TPDA & $30,36 \mathrm{ab}$ & $2,31 \mathrm{~b}$ & $1,18 \mathrm{~b}$ & $0,79^{\mathrm{ns}}$ & $1,06 \mathrm{ab}$ & $3,04 \mathrm{~b}$ & $112,94 \mathrm{~b}$ & $0,20 \mathrm{~b}$ \\
\hline & TPDM & $19,06 \mathrm{c}$ & $2,55 \mathrm{ab}$ & $1,25 \mathrm{~b}$ & $0,74^{\mathrm{ns}}$ & $0,81 \mathrm{~b}$ & $3,97 \mathrm{ab}$ & $100,09 \mathrm{~b}$ & $0,36 \mathrm{a}$ \\
\hline & TMDA & $34,55 \mathrm{a}$ & $2,70 \mathrm{a}$ & $1,92 \mathrm{a}$ & $0,97^{\mathrm{ns}}$ & $1,38 \mathrm{a}$ & $4,28 \mathrm{a}$ & $205,14 \mathrm{a}$ & $0,29 \mathrm{ab}$ \\
\hline & TMDM & $28,18 \mathrm{~b}$ & $2,58 \mathrm{ab}$ & $1,78 \mathrm{a}$ & $0,89^{\mathrm{ns}}$ & $1,30 \mathrm{a}$ & $3,97 \mathrm{ab}$ & $148,14 \mathrm{ab}$ & $0,31 \mathrm{a}$ \\
\hline \multirow[t]{4}{*}{110} & TPDA & $40,88 \mathrm{~b}$ & $2,81 \mathrm{~b}$ & $1,63 \mathrm{~b}$ & $1,09 \mathrm{~b}$ & $1,22 \mathrm{bc}$ & $3,94 \mathrm{~b}$ & $163,28 \mathrm{~b}$ & $0,23 \mathrm{~b}$ \\
\hline & TPDM & $34,17 \mathrm{c}$ & $2,87 \mathrm{~b}$ & $1,75 \mathrm{~b}$ & $0,85 \mathrm{~b}$ & $1,01 \mathrm{c}$ & $3,62 \mathrm{~b}$ & $140,47 \mathrm{~b}$ & $0,25 \mathrm{~b}$ \\
\hline & TMDA & $49,06 \mathrm{a}$ & $3,37 \mathrm{a}$ & $2,71 \mathrm{a}$ & $1,76 \mathrm{a}$ & $1,59 \mathrm{a}$ & $6,05 \mathrm{a}$ & $300,04 \mathrm{a}$ & $0,35 \mathrm{a}$ \\
\hline & TMDM & $38,25 \mathrm{bc}$ & $3,18 \mathrm{ab}$ & $2,67 \mathrm{a}$ & $1,40 \mathrm{ab}$ & $1,41 \mathrm{ab}$ & $5,48 \mathrm{ab}$ & $202,61 \mathrm{~b}$ & $0,37 \mathrm{a}$ \\
\hline \multirow[t]{4}{*}{125} & TPDA & $51,21 b$ & $3,05 \mathrm{~b}$ & $1,88 \mathrm{c}$ & $1,52 \mathrm{~b}$ & $1,43 \mathrm{~b}$ & $4,82 \mathrm{~b}$ & $190,97 \mathrm{~b}$ & $0,25 \mathrm{c}$ \\
\hline & TPDM & $43,84 \mathrm{c}$ & $3,18 \mathrm{~b}$ & $2,00 \mathrm{c}$ & $1,37 \mathrm{~b}$ & $1,32 \mathrm{~b}$ & $4,69 \mathrm{~b}$ & $182,84 \mathrm{~b}$ & $0,28 \mathrm{c}$ \\
\hline & TMDA & $62,25 \mathrm{a}$ & $3,68 \mathrm{a}$ & $3,06 \mathrm{~b}$ & $2,51 \mathrm{a}$ & $2,09 \mathrm{a}$ & $7,64 \mathrm{a}$ & $342,88 \mathrm{a}$ & $0,39 \mathrm{~b}$ \\
\hline & TMDM & $51,17 \mathrm{~b}$ & $3,68 \mathrm{a}$ & $3,60 \mathrm{a}$ & $2,48 \mathrm{a}$ & $2,23 \mathrm{a}$ & $8,30 \mathrm{a}$ & $337,95 \mathrm{a}$ & $0,49 \mathrm{a}$ \\
\hline \multirow[t]{4}{*}{140} & TPDA & $57,22 b$ & $3,43 \mathrm{~b}$ & $2,01 \mathrm{~b}$ & $2,05 \mathrm{c}$ & $1,65 \mathrm{~b}$ & $5,70 \mathrm{~b}$ & $192,67 \mathrm{~b}$ & $0,30 \mathrm{~b}$ \\
\hline & TPDM & $46,57 \mathrm{c}$ & $3,42 \mathrm{~b}$ & $2,22 \mathrm{~b}$ & $1,60 \mathrm{c}$ & $1,59 \mathrm{~b}$ & $5,39 \mathrm{~b}$ & $239,91 \mathrm{~b}$ & $0,36 \mathrm{~b}$ \\
\hline & TMDA & $66,04 \mathrm{a}$ & $4,19 \mathrm{a}$ & $3,62 \mathrm{a}$ & $4,02 \mathrm{a}$ & $2,52 \mathrm{a}$ & $10,15 \mathrm{a}$ & $362,27 \mathrm{a}$ & $0,54 \mathrm{a}$ \\
\hline & TMDM & $57,21 \mathrm{~b}$ & $4,08 \mathrm{a}$ & $3,63 \mathrm{a}$ & $3,31 \mathrm{~b}$ & $2,49 \mathrm{a}$ & $9,42 \mathrm{a}$ & $381,93 \mathrm{a}$ & $0,56 \mathrm{a}$ \\
\hline
\end{tabular}

*: significativo a $5 \%$ de probabilidade; ns: não significativo a 5\% de probabilidade; TPDA: tubete pequeno + densidade alta; TPDM: tubete pequeno + densidade média; TMDA: tubete médio + densidade alta; TMDM: tubete médio + densidade média.

Resultados semelhantes em altura, diâmetro de colo e massa seca total foram encontrados por Carneiro (1995) e Santos (2000), trabalhando com mudas de Pinus taeda e Cryptomeria japonica, respectivamente. Esses autores concluíram que mudas maiores são obtidas nos recipientes de dimensões maiores, pelo fato de haver um maior crescimento do sistema radicular e, consequentemente, um maior ganho em biomassa seca das mudas, fato este também verificado por Lopes (2005). Para Bonfim et al. 
(2009) tubetes com $50 \mathrm{~cm}^{3}$ produziram mudas que apresentaram resultados inferiores em relação aos recipientes com $288 \mathrm{~cm}^{3}$ e $577 \mathrm{~cm}^{3}$, sendo portanto inadequados para a produção de mudas.

A altura da parte aérea é considerada como um dos parâmetros mais antigos na classificação e seleção de mudas (PARVIAINEN, 1981), e ainda continua apresentando uma contribuição relativa importante, podendo ser indicada como um parâmetro para essa avaliação. Por ser uma variável de fácil medição e não ser um método destrutivo, sempre foi utilizada com eficiência para estimar o padrão de qualidade de mudas nos viveiros (GOMES et al., 2002), sendo considerada também como um dos mais importantes parâmetros para estimar o crescimento no campo (MEXAL; LANDS, 1990; REIS et al., 1991), além de sua medição não acarretar a destruição das mudas, o que a torna tecnicamente aceita como uma boa medida do potencial de desempenho (MEXAL; LANDS, 1990).

O IQD é apontado como bom indicador de qualidade de mudas porque são utilizados para seu cálculo a robustez (relação H/DC) e o equilíbrio da distribuição da biomassa (relação MSPA/MSR) (CALDEIRA et al., 2005; CALDEIRA et al., 2007), ponderando os resultados de várias características morfológicas importantes empregadas para avaliação da qualidade. Quanto maior o IQD, melhor é a qualidade da muda produzida (CALDEIRA et al., 2012).

A literatura evidencia que o IQD é uma característica variável (CALDEIRA et al., 2007; CALDEIRA et al., 2008 ; CALDEIRA et al., 2008b; SAIDELLES et al., 2009; TRAZZI et al., 2010). Assim, pode-se concluir que o IQD pode variar em função da espécie, do manejo das mudas no viveiro, do tipo e proporção do substrato, do volume do recipiente e, principalmente, de acordo com a idade em que a muda foi avaliada (GOMES et al., 2013).

Os resultados apresentados na tabela 2 relatam a tendência crescente do índice de qualidade Dickson ao longo do período de análise das mudas em viveiro para os quatro tratamentos. Observou-se, de maneira geral, que as mudas presentes no tratamento TMDM que apresentavam recipientes com maior volume de substrato explorável $\left(90 \mathrm{~cm}^{3}\right)$ e menor densidade das mudas na bandeja obtiveram um maior índice de qualidade Dickson, diferenciando-se significativamente dos outros tratamentos (Figura 1). Resultados similares foram relatados por Malavasi e Malavasi (2006), que verificaram em mudas de Cordia trichotoma e Jacaranda micranta produzidas em tubetes com capacidade volumétrica de 120, 180 e $300 \mathrm{~cm}^{3}$, valores médios de índice de qualidade Dickson estatisticamente iguais, e superiores aos das mudas produzidas nos tubetes de $55 \mathrm{~cm}^{3}$.

Da mesma forma, observou-se característica semelhante às variáveis área foliar, massa seca total e diâmetro de colo aos 140 DAE (Figura 1). Esses resultados corroboram trabalho realizado por Leles et al. (2006), em que mudas de Anadenanthera macrocarpa, Schinus terebinthifolius, Cedrela fissilis, Chorisia speciosa produzidas nos tubetes de maiores volumes apresentaram maior área foliar e maiores valores de índice de qualidade de Dickson.

O fato de a massa seca da raiz e do diâmetro de colo não apresentarem diferenças significativas em relação aos diferentes dias após a emergência e nos quatro tratamentos testados, apesar desta característica ser verificada no peso de matéria seca da parte aérea das mudas produzidas nos diferentes tamanhos de tubetes, pode ser explicado, de acordo com Reis et al. (1989), pelo ajuste de crescimento das mudas, no qual a restrição imposta pelo recipiente promove o crescimento balanceado entre as partes, sem alteração na distribuição relativa de matéria seca com a variação do volume do tubete. José et al. (2005) também não constataram diferenças significativas dessa relação de mudas de aroeira produzidas em volumes de tubetes de 50 e $150 \mathrm{~cm}^{3}$.

De acordo com José et al. (2005), normalmente mudas produzidas em condições de restrição radicular, como em tubetes de menor volume, passam por um processo de aclimatação, que pode propiciar o desenvolvimento de mecanismos de tolerância às condições de campo, podendo contribuir para o aumento no desempenho pós-plantio.

A partir da análise de correlação de Pearson apresentado na tabela 3, pode-se observar que todos os coeficientes das variáveis estudadas nos diferentes tratamentos testados apresentaram-se como significativos. Para alguns desses coeficientes, já eram esperados os resultados, como, por exemplo, as relações entre altura e diâmetro, dias após emergência, e as demais variáveis e as relações entre as massas secas.

A variável altura apresentou um índice de correlação relativamente alto quando relacionado com dias após emergência (Tabela 3). Essa afirmação é explicada pela competição entre mudas na bandeja, que, com o decorrer do tempo, influenciado pelo tamanho do tubete e pela densidade de mudas na bandeja, apresentou uma maior uniformidade com o espaço útil no crescimento para cada período em cada tratamento. 
Tabela 3. Coeficiente de correlação de Pearson entre as variáveis dias após a emergência (DAE), altura (H), em cm, diâmetro do colo (DC), em mm, massa seca de folhas (MSF), em g, massa seca do caule (MSC), em g, massa seca de raiz (MSR), em g, massa seca total (MST), em g, área foliar (AF), em $\mathrm{cm}^{2}$, e índice de qualidade de Dickson (IQD), em mudas de Eucalyptus grandis.

Table 3. Pearson correlation coefficient among the variables days after emergence (DAE), height $(\mathrm{H})$, in $\mathrm{cm}$, diameter (DC), in mm, leaf dry mass (MSF), in $\mathrm{g}$, the dry stem (MSC), in g, root dry weight (MSR), in g, total dry weight (MST), in g, leaf area (AF), in $\mathrm{cm}^{2}$, and quality index of Dickson (IQD) in Eucalyptus grandis seedlings.

\begin{tabular}{|c|c|c|c|c|c|c|c|c|c|}
\hline Tratamento & & $\mathbf{H}$ & DC & MSF & MSC & MSR & MST & $\mathbf{A F}$ & IQD \\
\hline \multirow[t]{8}{*}{ TPDA } & DAE & 0,96 & 0,95 & 0,90 & 0,85 & 0,88 & 0,93 & 0,85 & 0,88 \\
\hline & $\mathrm{H}$ & & 0,97 & 0,93 & 0,90 & 0,84 & 0,95 & 0,91 & 0,85 \\
\hline & $\mathrm{DC}$ & & & 0,96 & 0,91 & 0,85 & 0,98 & 0,91 & 0,93 \\
\hline & MSF & & & & 0,92 & 0,80 & 0,98 & 0,94 & 0,94 \\
\hline & MSC & & & & & 0,72 & 0,95 & 0,91 & 0,88 \\
\hline & MSR & & & & & & 0,87 & 0,68 & 0,89 \\
\hline & MST & & & & & & & 0,91 & 0,96 \\
\hline & $\mathrm{AF}$ & & & & & & & & 0,83 \\
\hline \multirow[t]{8}{*}{ TPDM } & DAE & 0,95 & 0,92 & 0,93 & 0,90 & 0,94 & 0,79 & 0,87 & 0,66 \\
\hline & $\mathrm{H}$ & & 0,91 & 0,93 & 0,89 & 0,93 & 0,79 & 0,89 & 0,59 \\
\hline & $\mathrm{DC}$ & & & 0,91 & 0,87 & 0,88 & 0,82 & 0,88 & 0,83 \\
\hline & MSF & & & & 0,89 & 0,87 & 0,80 & 0,85 & 0,65 \\
\hline & MSC & & & & & 0,86 & 0,80 & 0,90 & 0,67 \\
\hline & MSR & & & & & & 0,82 & 0,87 & 0,67 \\
\hline & MST & & & & & & & 0,52 & 0,93 \\
\hline & $\mathrm{AF}$ & & & & & & & & 0,64 \\
\hline \multirow[t]{8}{*}{ TMDA } & DAE & 0,97 & 0,97 & 0,93 & 0,91 & 0,91 & 0,95 & 0,84 & 0,90 \\
\hline & $\mathrm{H}$ & & 0,96 & 0,92 & 0,92 & 0,89 & 0,94 & 0,85 & 0,86 \\
\hline & DC & & & 0,94 & 0,91 & 0,91 & 0,95 & 0,88 & 0,93 \\
\hline & MSF & & & & 0,90 & 0,91 & 0,97 & 0,89 & 0,95 \\
\hline & MSC & & & & & 0,89 & 0,97 & 0,83 & 0,91 \\
\hline & MSR & & & & & & 0,95 & 0,84 & 0,96 \\
\hline & MST & & & & & & & 0,88 & 0,97 \\
\hline & $\mathrm{AF}$ & & & & & & & & 0,87 \\
\hline \multirow[t]{8}{*}{ TMDM } & DAE & 0,94 & 0,93 & 0,91 & 0,78 & 0,84 & 0,87 & 0,86 & 0,84 \\
\hline & $\mathrm{H}$ & & 0,96 & 0,96 & 0,91 & 0,89 & 0,96 & 0,93 & 0,89 \\
\hline & DC & & & 0,97 & 0,92 & 0,88 & 0,96 & 0,93 & 0,95 \\
\hline & MSF & & & & 0,91 & 0,89 & 0,98 & 0,94 & 0,94 \\
\hline & MSC & & & & & 0,86 & 0,97 & 0,94 & 0,93 \\
\hline & MSR & & & & & & 0,94 & 0,88 & 0,94 \\
\hline & MST & & & & & & & 0,96 & 0,97 \\
\hline & $\mathrm{AF}$ & & & & & & & & 0,92 \\
\hline
\end{tabular}

* Todos os coeficientes de correlação foram significativos em 5\% de probabilidade de erro, pelo teste t. TPDA: tubete pequeno + densidade alta; TPDM: tubete pequeno + densidade média; TMDA: tubete médio + densidade alta; TMDM: tubete médio + densidade média.

A variável área foliar foi a que apresentou, para a maioria das relações, baixas estimativas do coeficiente de correlação, mesmo sendo significativos, mostrando que é arriscado utilizar essa variável como um indicador da qualidade de mudas. É importante ressaltar ainda que a avaliação da área foliar demanda muito tempo, tornando a análise inviável. 

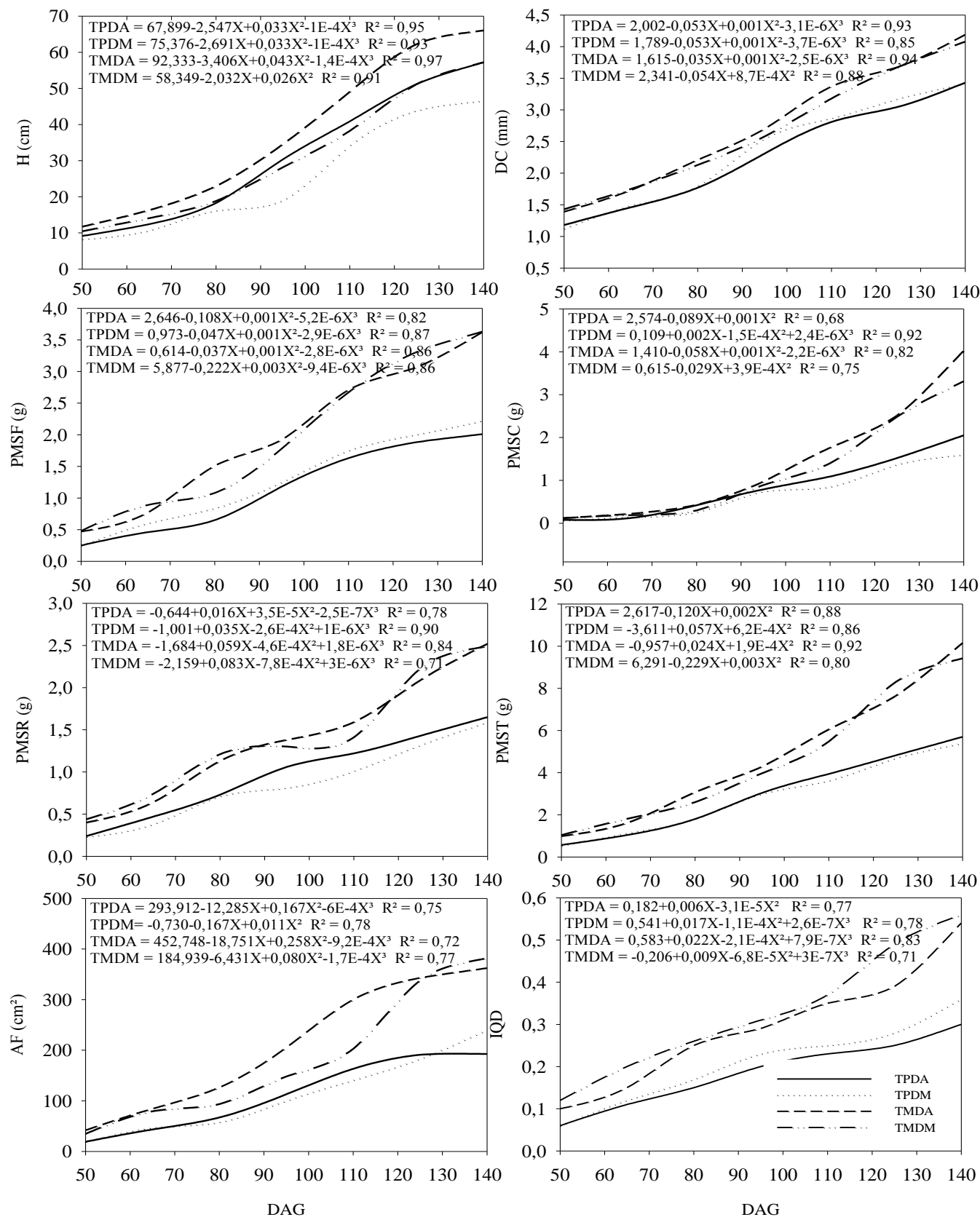

Figura 1. Modelos estatísticos para as variáveis dias após a emergência (DAE), altura (H), diâmetro do colo (DC), massa seca de folhas (MSF), massa seca do caule (MSC), massa seca de raiz (MSR), massa seca total (MST), área foliar (AF) e índice de qualidade de Dickson (IQD), nos diferentes tratamentos: tubete pequeno + densidade alta (TPDA), tubete pequeno + densidade média (TPDM), tubete médio + densidade alta (TMDA) e tubete médio + densidade média (TMDM), em mudas de Eucalyptus grandis.

Figure 1. Statistical models for the variables days after emergence (DAE), height (H), diameter (DC), leaf dry mass (MSF), stem dry mass (MSC), root dry weight (MSR), mass total dry matter (MST), leaf area (AF) and quality index of Dickson (IQD) for different treatments: small container + high density (TPDA), small container + average density (TPDM), container average + high density (TMDA) and container average + average density (TMDM) in Eucalyptus grandis. 
Pela análise de correlação de Pearson, a massa seca total se mostrou altamente relacionada com índice de qualidade de Dickson, diâmetro do colo, massa seca de folhas, massa seca de caule e massa seca de raízes, em ambos os tratamentos (Tabela 3). Fonseca et al. (2002), estudando a qualidade de mudas de Trema micrantha (L.) Blume, afirmam que o índice de qualidade de Dickson é altamente correlacionado com todos os parâmetros morfológicos da planta. Obviamente, as relações entre o índice de qualidade de Dickson e as massas já eram esperadas, devido a estas serem utilizadas na fórmula de cálculo daquele, sendo também variáveis com risco no indicativo da qualidade de muda, também devido à característica de perdas das mudas ao se avaliarem tais variáveis.

José et al. (2005), pesquisando a produção de mudas de aroeira (Schinus terebinthifolius Raddi) para recuperação de áreas degradadas, verificaram que o potencial de crescimento do sistema radicular apresentou correlação significativa com outras variáveis, no entanto, foi com o diâmetro do colo a sua resposta mais evidente. O diâmetro do colo foi a característica que mais se correlacionou com as outras variáveis, como também afirmam Mexal e Landis (1990), Brisset et al. (1991), Johnson e Cline (1991) e José et al. (2005).

Deve ser dada importância ao sistema radicular de mudas, em relação ao estudo dos seus parâmetros morfológicos, assegurando melhor desempenho no campo. Assim, as raízes estão intimamente associadas às atividades de natureza fisiológica das mudas, no complexo ambiente-solo-água-planta (CARNEIRO, 1995), concordando com Hermann (1964), na sua afirmativa de que o peso de matéria seca das raízes é reconhecido como um dos melhores e mais importantes parâmetros para a sobrevivência e estabelecimento das mudas em campo. Em mudas de Pseudotsuga menziesii, a sobrevivência foi consideravelmente maior quanto mais abundante foi o sistema radicular, independentemente da altura da parte aérea (HERMANN, 1964).

Um índice eficiente e seguro para avaliar a qualidade de mudas pode ser a relação do peso de matéria seca da parte aérea/peso de matéria seca das raízes (PARVIAINEM, 1981), sendo positivamente verificada essa relação pela tabela 3, quando analisado o coeficiente de correlação de Pearson da massa seca de caule e da massa seca de folhas com a fitomassa seca de raiz.

A produção de matéria seca tem sido considerada um dos melhores parâmetros para caracterizar a qualidade de mudas, apresentando, porém, o inconveniente de não ser viável a sua determinação em muitos viveiros, principalmente por envolver a destruição completa da muda e a utilização de estufas.

\section{CONCLUSÕES}

- $\quad$ O índice de qualidade de Dickson é altamente correlacionado com a massa seca total, sendo que os tratamentos TMDA e TMDM, que apresentam tubetes médios, resultaram nos melhores índices de qualidade Dickson, produzindo mudas com boa qualidade.

\section{REFERÊNCIAS}

BOMFIM, A. A.; NOVAES, A, B.; JOSÉ, A. R. S.; GRISI. F. A. Avaliação morfológica de mudas de madeira-nova (Pterogyne nitens Tull.) produzidas em tubetes e sacos plásticos e de seu desempenho no campo. Floresta, Curitiba, v. 39, n. 1, p. 33 - 40, 2009.

BRISSET, J. C.; BARNETT, J. P.; LANDIS, T. D. Container seedlings. In: DURYEA, M. L.; DOUGHERTY, P. M. (Eds.). Forest regeneration manual. Netherlands: Klumer Academic, 1991. p. $117-142$.

CALDEIRA, M. V. W.; BLUM, H.; BALBINOT, R.; LOMBARDI, K. C. Uso do resíduo do algodão no substrato para produção de mudas florestais. Revista Acadêmica: Ciências Agrárias e Ambientais, v. 6, p. 191 - 202. 2008a.

CALDEIRA, M. V. W.; DELARMELINA, W. M.; LÜBE, S. G.; GOMES, D. R.; GONÇALVES, E. O.; ALVES, A. F. Biossólido na composição de substrato para a produção de mudas de Tectona grandis. Floresta, v. 42, n. 1, p. 77 - 84, 2012.

CALDEIRA, M. V. W.; MARCOLIN, M.; MORAES, E.; SCHAADT, S. S. Influência do resíduo da indústria do algodão na formulação de substrato para produção de mudas de Schinus terebinthifolius Raddi, Archontophoenix alexandrae Wendl. et Drude e Archontophoenix cunninghamiana Wendl. et Drude. Ambiência, Guarapuava, v. 3, p. 1 - 8, 2007. 
CALDEIRA, M. V. W.; ROSA, G. N.; FENILLI, T. A. B.; HARBS, R. M. P. Composto orgânico na produção de mudas de aroeira-vermelha. Scientia Agraria, v. 9, p. 27 - 33. 2008 b.

CALDEIRA, M. V. W.; SPATHELF, P.; BARICHELLO, L. R.; VOGEL, H. L. M.; SCHUMACHER, M. V. Effect of different doses of vermicompost on the growth of Apuleia leiocarpa (Vog) Macbr. seedlings. Revista Acadêmica: Ciências Agrárias e Ambientais, v. 3, p. 11 - 17, 2005.

CARNEIRO, J. G. A. Produção e controle de qualidade de mudas florestais. Curitiba: Campos/UENF. UFPR/FUPEF, 1995. $451 \mathrm{p}$.

CARON, B. O.; SOUZA, V. Q.; TREVISAN, R.; BEHLING, A.; SCHMIDT, D.; BAMBERG, R.; ELOY, E. Eficiência de conversão da radiação fotossinteticamente ativa interceptada em fitomassa de mudas de eucalipto. Revista Árvore, v. 36, p. 833 - 842, 2012.

CRUZ, C. A.; PAIVA, H. N.; GOMES, K. C. O.; GUERRERO, C. R. A. Efeito de diferentes níveis de saturação por bases no desenvolvimento e qualidade de mudas de ipê-roxo (Tabebuia impetiginosa (Mart.) Standley). Scientia Forestalis, n. 66, p. 100 - 107, 2004.

DICKSON, A.; LEAF, A. L.; HOSNER, J. F. Quality appraisal of white spruce and white pine seedling stock in nurseries. Forestry Chronicle, v. 36, p. 10 - 13, 1960.

FONSECA, E. P.; VALERI, S. V.; MIGLIORANZA, E.; FONSECA, N. A. N.; COUTO, L. Padrão de Qualidade de mudas de Trema micrantha (L.) Blume., produzidas sob diferentes períodos de sombreamento. Revista Árvore, v. 26, n. 4, p. 515 - 523, 2002.

GOMES, D. R.; CALDEIRA, M. V. W.; DELARMELINA, W. M.; GONÇALVES, E. O.; TRAZZI, P. A. Lodo de esgoto como substrato para a produção de mudas de Tectona grandis L. Cerne, v. 19, n. 1, p. $123-131,2013$.

GOMES, J. M.; COUTO, L.; LEITE, H. G.; XAVIER, A.; GARCIA, S. L. R. Parâmetros morfológicos na avaliação da qualidade de mudas de Eucalyptus grandis. Revista Árvore, Viçosa, v. 26, n. 6, p. 655 664, 2002.

HERMANN, R. K. Importance of top-root ratios for survival of Douglas-fir seedlings. Tree Planters Notes, v. 64, 7 - 11. 1964.

JOHNSON, J. D.; CLINE, M. L. Seedling quality of southern pines. In: DURYEA, M. L.; DOUGHERTY, P. M. (Eds.). Forest regeneration manual. Dordrecht Netherlands: Klumer Academic Publishers, 1991. p. 143 - 162.

JOSÉ, A. C.; DAVIDE, A. C.; OLIVEIRA, S. L. Produção de mudas de aroeira (Schinus terebinthifolius Raddi) para recuperação de áreas degradadas pela mineração de bauxita. Cerne, v. 11, n. 2, p. 187 - 196, 2005.

LELES, P. S. S.; LISBOA, A. L.; OLIVEIRA NETO, S. N.; GRUGIKI, M. A.; FERREIRA, M. A. Qualidade de mudas de quatro espécies florestais produzidas em diferentes tubetes. Floresta e Ambiente, v. 13, n. 1, p. 69 - 78, 2006.

MAFIA, R. G.; ALFENAS, A. C.; SIQUEIRA, L.; LEITE, H. G.; CAVALLAZZI, J. R. P. Critério técnico para determinação da idade ótima de mudas de eucalipto para plantio. Revista Árvore, v. 29, n. 6, p. $947-953,2005$.

MALAVASI, U. C.; MALAVASI, M. M. Efeito do volume do tubete no crescimento inicial de plântulas de Cordia trichotoma (Vell.) Arrab. ex Steud e Jacaranda micranta Cham. Ciência Florestal. v. 16, n. 1, p. $11-16,2006$.

MEXAL, J. L.; LANDIS, T. D. Target seedling concepts: height and diameter. In: TARGET SEEDLING SYMPOSIUM, MEETING OF THE WESTERN FOREST NURSERY ASSOCIATIONS, GENERAL TECHNICAL REPORT RM-200, 1990, Roseburg. Proceedings... p. 17 - 35. Fort. Collins: United States Department of Agriculture, Forest Service, 1990. 
PARVIAINEN, J. V. Qualidade e avaliação da qualidade de mudas florestais. In: SEMINÁRIO DE SEMENTES E VIVEIROS FLORESTAIS, 1., 1981, Curitiba. Anais do... Curitiba: FUPEF, 1981. p. 59 90.

REIS, E. R.; LÚCIO, A. D. C; FORTES, F. O.; LOPES, S. J.; SILVEIRA, S. D. Período de permanência de mudas de Eucalyptus grandis em viveiro baseado em parâmetros morfológicos. Revista Árvore, Viçosa, v. 32, n. 5, p. 809 - 814. 2008.

REIS, G. G.; REIS, M. G. F.; MAESTRI, M. Crescimento de Eucalyptus camaldulensis, Eucalyptus grandis e Eucalyptus cloeziana sob diferentes níveis de restrição radicular. Revista Árvore, v. 13, n. 1, p. 1 - 18, 1989.

REIS, M. G. F.; REIS, G. G.; REGAZZI, A. J.; LELES, P. S. S. Crescimento e forma de fuste de mudas de jacarandá-da-bahia (Dalbergia nigra Fr. Allem.) sob diferentes níveis de sombreamento e tempo de cobertura. Revista Árvore, v. 15, n. 1, p. 23 - 34, 1991.

SAIDELLES, F. L. F.; CALDEIRA, M. V. W.; SCHIRMER, W. N.; SPERANDIO, H. V. Casca de arroz carbonizada como substrato para produção de mudas de tamboril-da-mata e garapeira. Semina: Ciências Agrárias, v. 30, p. 1173 - 1186. 2009.

SANTOS, C. B. Efeito do volume de tubetes e tipos de substratos na qualidade de mudas de Cryptomeria japonica. Ciência Florestal, v. 10, n. 2, p. 1 - 15, 2000.

SAS LEARNING EDITION. Getting started with the SAS learning edition. Cary, 2003. 200 p.

TEIXEIRA, P. C.; RODRIGUES, H. S.; LIMA, W. A. A. de; ROCHA, R. N. C. da; CUNHA, R. N. V. da; LOPES, R. Influência da disposição dos tubetes e da aplicação de fertilizantes de liberação lenta, durante o pré-viveiro, no crescimento de mudas de dendezeiro (Elaeis guineensis Jacq.). Ciência Florestal, v. 19, n. 2, p. 157 - 168, 2009.

TRAZZI, P. A.; CALDEIRA, M. V. W.; COLOMBI, R. Avaliação de mudas de Tecoma stans utilizando biossólido e resíduo orgânico. Revista de Agricultura, Piracicaba, v. 85, p. 218 - 226, 2010. 
